\title{
Correction to: Membership Criteria and Containments of Powers of Monomial Ideals
}

\author{
Huy Tài Hà ${ }^{1} \cdot$ Ngo Viet Trung ${ }^{2}$
}

Received: 5 July 2021 / Published online: 23 August 2021

(C) Institute of Mathematics, Vietnam Academy of Science and Technology (VAST) and Springer Nature Singapore Pte Ltd. 2021

\section{Correction to: Acta Mathematica Vietnamica https://doi.org/10.1007/s40306-018-00325-y}

This corrigendum provides a correction to the definition of $\rho_{\text {inf }}$ which was given in $[1, \mathrm{p}$. 128]. The correct definition of this invariant is as follows.

Definition 0.1 Let $I$ be a homogeneous ideal. Set

$$
\rho_{\text {inf }}(I)=\inf \left\{q \mid I^{(h)} \subseteq I^{k} \text { whenever } \frac{h}{k} \geq q\right\} .
$$

Recall that

$$
\rho(I)=\sup \left\{\frac{h}{k} \mid I^{(h)} \nsubseteq I^{k}\right\} .
$$

With this definition of $\rho_{\text {inf }}(I)$, the observation that $\rho(I) \leq \rho_{\text {inf }}(I)$ on $[1$, p. 128] and [1, Corollary 3.6] are true as stated.

Acknowledgements We thank Brian Harbourne and Arvind Kumar for pointing out the mistake in our original definition of $\rho_{\text {inf }}$.

The original article can be found online at https://doi.org/10.1007/s40306-018-00325-y.

Ngo Viet Trung

nvtrung@math.ac.vn

Huy Tài Hà

tha@tulane.edu;

http://www.math.tulane.edu/ tai/

1 Department of Mathematics, Tulane University, 6823 St. Charles Ave., New Orleans, LA 70118, USA

2 International Centre for Research and Postgraduate Training, Institute of Mathematics, Vietnam Academy of Science and Technology, 18 Hoang Quoc Viet, Hanoi, Vietnam 


\section{References}

1. Hà, H.T., Trung, N.V.: Membership criteria and containments of powers of monomial ideals. Acta Math. Vietnam. 44, 117-139 (2019)

Publisher's Note Springer Nature remains neutral with regard to jurisdictional claims in published maps and institutional affiliations. 